\title{
Optical properties and structure of $\mathrm{HfO}_{2}$ thin films grown by high pressure reactive sputtering
}

\author{
F. L. Martínez, ${ }^{1}$ M. Toledano-Luque, ${ }^{2}$ J. J. Gandía, ${ }^{3}$ J. Cárabe, ${ }^{3}$ W. Bohne, ${ }^{4}$ J. Röhrich, ${ }^{4}$ E. \\ Strub, ${ }^{4}$ I. Mártil ${ }^{2}$
}

${ }^{1}$ Departamento de Electrónica y Tecnología de Computadoras, Universidad Politécnica de Cartagena, Campus Universitario Muralla del Mar, E-30202 Cartagena, Spain.

${ }^{2}$ Departamento de Física Aplicada III, Universidad Complutense de Madrid, E-28040 Madrid, Spain.

${ }^{3}$ Departamento de Energía, CIEMAT, Avda. Complutense 22, E-28040 Madrid, Spain.

${ }^{4}$ Hahn-Meitner-Institut Berlin, Abteilung SF4, D-14109 Berlin, Germany.

\begin{abstract}
Thin films of hafnium oxide have been grown by high pressure reactive sputtering on transparent quartz substrates (UV-grade silica) and silicon wafers. Deposition conditions were adjusted to obtain polycrystalline as well as amorphous films. Optical properties of the films deposited on the silica substrates were investigated by transmittance and reflectance spectroscopy in the ultraviolet, visible and near infrared range (UV-VIS-NIR). A numerical analysis method that takes into account the different surface roughness of the polycrystalline and amorphous films was applied to calculate the optical constants (refractive index and absorption coefficient). Amorphous films were found to have a higher refractive index and a lower transparency than polycrystalline films. This is attributed to a higher density of the amorphous samples, which was confirmed by atomic density measurements performed by heavy-ion elastic recoil detection analysis (ERDA). The absorption coefficient gave an excellent fit to the Tauc law (indirect gap), which allowed to obtain a band gap value of 5.54 $\mathrm{eV}$. The structure of the films (amorphous or polycrystalline) was found to have no significant influence on the nature of the band gap. The Tauc plots also give information about the structure of the films, because the slope of the plot (the Tauc parameter) is related to the degree of order in the bond network. The amorphous samples had a larger value of the Tauc parameter, i.e., more order than the polycrystalline samples. This is indicative of a uniform bond network with percolation of the bond chains, in contrast to the randomly oriented polycrystalline grains separated by grain boundaries.
\end{abstract}




\section{Introduction.}

The electrical properties of hafnium oxide and other metallic oxides with high dielectric constants are being currently investigated with great interest, specially due to the promising possibilities that these materials have found for replacing silicon oxide at the heart of MOS (metal-oxide-semiconductor) transistors [1, 2]. Over the past years plenty of work has been done with several dielectric materials such as silicon nitrides $[3,4,5,6]$ and oxynitrides [7, 8, 9], while nitridation of the oxide layer has been used by the semiconductor industry to reduce tunneling currents through the gate dielectric [10]. However, at the present scale of integration (65 nm process generation), the oxynitride has to be so thin (less than 1.7 $\mathrm{nm}$ ) that tunneling currents impose severe limitations to the performance of the transistors [10]. A dielectric with higher permittivity than silicon oxynitride could be made thicker and still have the same capacitance as an equivalent oxide with a proportionally lower thickness, where the proportionality constant is the ratio of the permittivities of the two materials.

Hence, a more aggressive downscaling of the transistor dimensions will require dielectrics of even higher permittivities. Some transition metal oxides and silicates, specially from groups IV and V (Ti, Hf, Ta), have attracted considerable attention, and most efforts are now concentrated in solving the mobility degradation problems [11] associated with the higher polarization characteristics of these materials. Trapping of charge due to bulk traps [12], with the consequent shift in threshold voltage during device operation, and Fermi-level pinning caused by a high density of interface states, are also common problems of high-k dielectrics $[1,2,10]$. Concerning process stability, the most promising among the high-k dielectrics is hafnium oxide $\left(\mathrm{HfO}_{2}\right)$ [13], which has a suitable conduction band offset with silicon [13], so we have selected this material for our present study. As deposition method we took an innovative approach, selecting high pressure reactive sputtering (HPRS) $[14,15]$ for its potential advantages for low interfacial damage compared with other plasma processes. Due to the higher deposition pressure, sputtered species have a shorter mean free path length than the distance from target to substrate. Therefore, the sputtered particles are thermalized and their energy when reaching the substrate is moderated, reducing the generation of defects at the interface.

As most present studies are driven by the urgency of the industry to find a solution for the gate dielectric problem, not so much emphasis has been placed in investigating the properties of these materials by optical methods. In some cases, spectroscopic ellipsometry has been applied to determine the optical constants of $\mathrm{HfO}_{2}[16,17]$ and to relate bulk defects with electrical characteristics [18]. Raman active phonons have been investigated both 
theoretically [19, 20] and experimentally [20, 21], but always in crystals prepared in bulk form. Infrared active phonons have also been calculated [19] and measured [22, 23], but unlike Raman spectra, the infrared absorption peaks can be observed in thin film samples. In spite of the relatively scarce literature about its optical properties, hafnium oxide has interesting optical applications. For example, due to the large band gap and high refractive index it has been used as optical coatings for astronomical CCDs [24] and as antireflective multi-layer coatings for night vision devices and for IR optical devices [25].

Ultraviolet-visible spectroscopy in the region around the optical gap can be used to determine the value of the band gap. For this purpose, one recent publication [26] uses transmittance spectra of samples deposited on transparent substrates, while another study measures reflectance [27] of samples deposited on silicon. However, there are no published optical constants of $\mathrm{HfO}_{2}$ determined from the simultaneous analysis of transmittance (T) and reflectance (R) spectra. The use of both $\mathrm{T}$ and $\mathrm{R}$ allows for a much more accurate determination of the optical absorption coefficient $(\alpha)$ in the region around the optical gap, because a numerical analysis method can be implemented that solves simultaneously the equations of $\mathrm{T}$ and $\mathrm{R}$ as a function of wavelength $\lambda$, where the complex refractive index of the thin film layer appears as a parameter and is determined by fitting its real part to a Cauchy dispersion law [28, 29].

The aim of this study is to use this method of analysis for the ultraviolet-visible-near infrared spectra of $\mathrm{HfO}_{2}$ thin films deposited on transparent quartz substrates (UV-grade silica). For this purpose, transmittance and reflectance spectra are taken in the whole range from $200 \mathrm{~nm}$ to $2000 \mathrm{~nm}$ and are analyzed simultaneously by a numerical method that also takes into account the scattering caused by the surface roughness of the samples [28, 29]. This is specially important because two different kinds of samples with very different morphological characteristics are analyzed in this study. These two types of samples differ in their crystalline structure: one is amorphous and the other polycrystalline. By adjusting the composition of the constituent gas of the plasma discharge of our HPRS system, we can modify the structure of the films. We demonstrate these differences by X-ray diffraction (XRD) and by Fourier transform infrared spectroscopy (FTIR), as well as by measurements of the atomic area density with heavy-ion Elastic Recoil Detection Analysis (ERDA). Finally, we show capacitance measurements versus polarization $(C-V)$, and the density of interface states that can be extracted from these curves, which reveal the consequences that the change in the structure of the film has for its electrical characteristics. 


\section{Experimental procedure.}

The substrates for the growth of the $\mathrm{HfO}_{2}$ thin films were selected according to each characterization technique. For the optical measurements we chose UV-grade quartz (silica), whose transparency range extends down to $190 \mathrm{~nm}$ and covers the absorption edge of the hafnium oxide. In the case of the samples for XRD, ERDA and electrical measurements, we used silicon substrates of low resistivity $(5 \Omega \mathrm{cm})$, while for FTIR characterization it was necessary to use high resistivity ( $80 \Omega \mathrm{cm}$ ) silicon in order to minimize free-electron absorption. Every type of substrate was subjected to an appropriate cleaning procedure (a standard RCA process [30] for silicon and organic solvents for quartz) before being introduced in the deposition chamber.

The deposition parameters of our HPRS system that result in optimum properties for the $\mathrm{HfO}_{2}$ films grown with $\mathrm{O}_{2}$ plasmas are known from our previous work [31]. An important result that we discovered recently is that in a pure Ar plasma the structure of the films becomes amorphous, while with pure $\mathrm{O}_{2}$ or mixed $\mathrm{O}_{2}$ /Ar plasmas it is polycrystalline. Hence, we used one process with pure $\mathrm{O}_{2}$ at a pressure of 1.2 mbar to grow the polycrystalline film, and another one with pure Ar at the same deposition pressure to grow the amorphous samples. The substrate temperature was $200{ }^{\circ} \mathrm{C}$ in both cases, while radio frequency (rf) power was 60 W. The $\mathrm{HfO}_{2}$ cathode had a purity of $99.95 \%$.

Deposition time was 30 minutes for all samples except those grown on quartz for the optical measurements, where longer deposition times were necessary to obtain a good absorption edge. The thickness was measured by cross-sectional transmission electron microscopy (TEM) photographs of samples deposited under the same conditions and

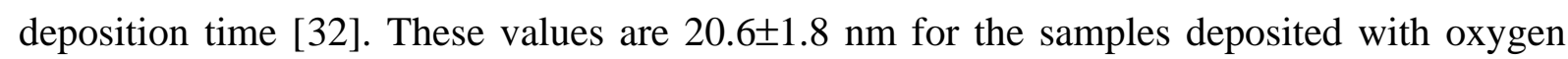
plasma and $10.9 \pm 0.3 \mathrm{~nm}$ for films grown in argon plasma (in both cases the deposition time is 30 minutes).

Some of the obtained dielectric films were analyzed by HI-ERDA to determine the atomic concentration of all constituent elements. In a previous report [31] we made use of these results to determine the stoichiometry and content of impurities. Here we will concentrate on the value of mass density that can be derived from the atomic area density and the thickness of the samples, and we will compare it with the optical density given by the refractive index. The HI-ERDA measurements were performed at the Ionen-Strahl-Labor (ISL) of the Hahn-Meitner-Institut in Berlin, using a beam of Au ions accelerated to an energy of $350 \mathrm{MeV}$. The recoiled atoms from the sample were detected by a mass and energy 
dispersive spectrometer [33]. The total ion dose was in the order of $10^{11}$ atoms, which results in a fluence of about $10^{12}$ atoms $/ \mathrm{cm}^{2}$. This is below a value that produces the preferential effusion effects of light atoms that we have studied in a previous report [34].

The structure of the films was investigated by glancing incidence X-ray diffraction spectroscopy ( $0.5^{\circ}$ incidence angle) and infrared spectroscopy. The X-ray diffractograms were obtained with a Philips XPERT diffractometer in the $\theta-2 \theta$ configuration using the $\mathrm{Cu} \mathrm{K}_{\alpha}$ line. IR spectra were taken with a Nicolet Magna-IR spectrometer in transmission mode and normal incidence. Both types of spectra revealed the differences between amorphous and polycrystalline films.

For the optical analysis we measured the transmittance and reflectance spectra in the ultraviolet-visible-near infrared range (UV-VIS-NIR) with a Perkin-Elmer spectrometer. While most published results measure only transmittance [26, 35] and calculate the complex refractive index by a method based on Swanepoel [36], we used a more innovative approach developed in our group, which involves reversed equations of transmittance and reflectance as a function of $n$ and $k$ (real and imaginary part, respectively, of the refractive index of the film), with the wavelength $(\lambda)$ as an independent variable. Two parameters appear in these equations and serve as fitting parameters: the thickness $(d)$ of the film and the coherence factor $(C)$ [28], which takes into account the loss of coherence during multiple reflection and how it affects the interference in the transparency region. This loss of coherence may be caused by surface roughness, which results in different path lengths of successively reflected photons. Reflections in random directions at grain boundaries or defects may have a similar effect. The evaluation procedure starts with trial values of $d$ and $C$. The equations are solved numerically and the resulting values of $n$ versus $\lambda$ are fitted by a Cauchy dispersion rule. The computer program then varies systematically the values of $d$ and $C$ until it obtains the best fit of $n$ to the Cauchy law. As a result, the imaginary part of the refractive index is also obtained, so the absorption coefficient can be calculated as $\alpha=4 \pi k / \lambda$. The values of thickness obtained by this method are $230 \mathrm{~nm}$ for the sample grown with oxygen plasma and $168 \mathrm{~nm}$ when argon plasma is used instead of oxygen.

The graphs of absorption coefficient versus photon energy $(h v)$ are studied to analyze the nature and value $\left(E_{\mathrm{G}}\right)$ of the band gap. A direct band gap follows the law:

$$
(\alpha h v)^{2}=A\left(h v-E_{G}\right),
$$


so, if there is a direct band gap, a plot of $(\alpha h v)^{2}$ versus $h v$ would have a linear region with slope $A$ and whose extrapolation to $\alpha(h v)=0$ would give the value of $E_{\mathrm{G}}$. On the other hand, data from indirect band gaps meet usually the Tauc law:

$$
\sqrt{\alpha h v}=B_{\text {Tauc }}\left(h v-E_{G}\right),
$$

where $B_{\text {Tauc }}$ (Tauc parameter) is the slope of the linear region in a plot of $(\alpha h v)^{1 / 2}$ versus $h v$, whose extrapolation to $\alpha(\mathrm{hv})=0$ would give the value of the indirect band gap. It should be noted that these relationships are valid only for parabolic bands, so they are only physically meaningful for values of photon energies not much larger than the band gap energy, for which the absorption is not very strong. They are not valid at photon energies lower than the band gap, where the absorption coefficient falls below $10^{-2}-10^{-1} \mu \mathrm{m}^{-1}$. In this range the absorption coefficient is an exponential rather than a potential function of energy, and it is given by the Urbach law:

$$
\alpha=\alpha_{0} e^{\frac{h v}{E_{0}}}
$$

where $E_{0}$ is called Urbach energy. This law accounts for an exponential tail in the absorption coefficient that is usually associated with localized band tail states. Hence, both the Tauc parameter and the Urbach energy are related to lattice order, because they depend on how steep is the increase of the absorption coefficient.

Electrical measurements were done on MOS capacitors with Al gate electrodes of $10^{-3}$ $\mathrm{cm}^{2}$ of area fabricated by e-beam evaporation. The $\mathrm{HfO}_{2}$ films in these structures were grown with the same conditions and deposition time as those analyzed by ERDA, XRD and FTIR, and therefore are expected to have the same thickness. The MOS capacitors were characterized by means of capacitance measurements by the high-low frequency method [37], where quasistatic and high frequency $(100 \mathrm{kHz})$ capacitance $\left(C_{\mathrm{Q}}\right.$ and $C_{\mathrm{HF}}$, respectively) are measured simultaneously as a function of bias voltage. This method allows for the determination of the energy distribution of the interface trap density $\left(D_{\mathrm{it}}\right)$, provided that the leakage current is negligible compared to the displacement current (a requisite that is met by the films analyzed in this study).

\section{Results.}

The X-ray diffractograms of Figure 1 demonstrate the important result that samples deposited in $\mathrm{O}_{2}$ plasma are polycrystalline while samples deposited in Ar plasma are amorphous. The reasons for this shall be discussed in the next section. Vertical lines in the 
figure mark the characteristic peaks of the $\mathrm{HfO}_{2}$ monoclinic phase [38]. Clearly, the sample deposited with $\mathrm{O}_{2}$ plasma presents all these diffraction peaks, while the sample deposited with Ar plasma only shows a broad weak band that reveals its amorphous structure. In a previous work we showed that mixed $\mathrm{O}_{2} /$ Ar plasmas with different gas flow ratios always formed films with a polycrystalline structure [32]. So, it is necessary to eliminate completely the presence of $\mathrm{O}_{2}$ to obtain amorphous films. The most intense of the diffraction peaks is the (111) feature, which can be used to estimate the average grain size from the full width at half maximum (FWHM) of a Gaussian fit to this peak, according to the Scherrer equation [39]. From this calculation we estimate an average grain size of $20 \mathrm{~nm}$ for the polycrystalline film.

The different structure between the two kinds of samples is also noticeable in the midinfrared spectra shown in Figure 2. Vertical lines mark the peaks associated with the $\mathrm{HfO}_{2}$ monoclinic form. In the mid-infrared region there is only one strong absorption peak at 512 $\mathrm{cm}^{-1}$ [23]. The peak at $748 \mathrm{~cm}^{-1}$ has also been studied previously and assigned to an $A_{\mathrm{u}}$ mode (atomic displacement parallel to the $c$ axis of the unit-cell) [22]. As can be seen in Figure 2 both peaks disappear in the sample deposited with pure Ar plasma, while they are present in the sample deposited with $\mathrm{O}_{2}$ or mixed $\mathrm{O}_{2} / \mathrm{Ar}$ plasmas [32]. This is in agreement with previous reports $[23,40]$ that showed only a broad band starting at $500 \mathrm{~cm}^{-1}$ and extending into the far infrared region for amorphous hafnium oxide. The study of Neumayer and Cartier [23] also shows how this broad band gradually give rise to the characteristic peaks of the monoclinic structure as the sample crystallizes when annealed at increasingly higher temperatures. Therefore, the characteristic peaks of the monoclinic structure for the sample deposited with $\mathrm{O}_{2}$ reveal the different structure of both types of samples. The peak at $647 \mathrm{~cm}^{-1}$ has also been reported for monoclinic $\mathrm{HfO}_{2}$ in the work of Neumayer and Cartier [23] while the peak at $610 \mathrm{~cm}^{-1}$ has been studied by T. Hirata [22] and decomposed in two $B_{\mathrm{u}}$ infrared modes (corresponding to atomic displacements parallel to the $a b$ plane of the unit cell). However, the infrared spectra studied by Hirata correspond to sintered pellets which were not affected by the absorption of bulk silicon phonons from the substrate. Other authors who have studied the infrared spectra of hafnium oxide deposited on silicon have ignored the region around $610 \mathrm{~cm}^{-1}$ because it coincides with the absorption of a strong Si phonon [40]. That applies also to our case and explains why it does not disappear in the amorphous sample, so we attribute this feature to incomplete subtraction of the silicon phonon. Finally, the feature at $680 \mathrm{~cm}^{-1}$ has been reported as characteristic of a $\mathrm{HfO}_{2}$ longitudinal-optical (LO) phonon mode that appears only at oblique incidence. That can not be the case in our measurements, since they are taken at normal incidence. The variable intensity with which this peak appears 
suggests that it is due to residual absorption of atmospheric $\mathrm{CO}_{2}$, which is known to occur at this frequency.

Transmittance and reflectance spectra of two samples are presented in Figure 3. The samples were deposited on transparent quartz substrates under the same conditions as those described for the XRD and FTIR studies. Obviously, the different structure of the two types of samples has also important consequences for their optical properties. As can be seen from Figure 3, the amorphous hafnium oxide has a lower transmittance and higher reflectance, which means that its index of refraction is higher than that for the polycrystalline sample. This implies that polycrystalline hafnium oxide has a lower packing density, which must be due to the empty voids at the interface between the grains. In comparison, the amorphous samples have neither grains nor discontinuities, so they present a continuous network of bonds that forms a more compact structure. From these spectra the real part of the index of refraction $(n)$ can be calculated and fitted to a Cauchy dispersion law according to the procedure described in the experimental section. Figure 4 shows the result of this calculation. As expected the amorphous film has a refractive index significantly higher than the polycrystalline sample. For comparison, the refractive index of bulk $\mathrm{HfO}_{2}$ at $632.8 \mathrm{~nm}$ is 2.08 [41], which is close to the value (1.98) of our amorphous samples at that wavelength.

In the calculation of the refractive index from the transmittance and reflectance spectra, not only the real part $n$ is obtained, but also the imaginary part or extinction coefficient $k$, from which the absorption coefficient can be calculated as $\alpha=4 \pi k / \lambda$. In Figure 5 we plot the absorption coefficient calculated in this way as a function of photon energy. Two regions can be distinguished: the absorption edge, which starts at around $5.6 \mathrm{eV}$, and the transparency range that extends below this energy. In the transparency region the polycrystalline sample has a higher absorption coefficient than the amorphous film. This is due to the loss of light by random reflections at the rough surface of the polycrystalline sample and at the interfaces between crystallites. This effect can also be seen in the transmittance and reflectance spectra of Figure 3, where the lower modulation of the interference oscillations in the spectra of the polycrystalline film is due to the loss of coherence of light during the multiple reflections. Hence, the lower transparency of the polycrystalline sample and the narrower oscillations of its interference pattern are both related to the loss of light by reflections in random directions at crystallites surfaces or interfaces. This demonstrates the advantage of our method for the analysis of the transmittance and reflectance spectra [28], that takes into account the loss of coherence of the light by introducing a coherence factor in the solution of the equations. 


\section{Discussion.}

The structural analysis of the hafnium oxide films by XRD and FTIR spectroscopy has shown that only a deposition with pure Ar plasma produces amorphous samples, while the introduction of any amount of oxygen in the plasma makes the film polycrystalline [32]. It is well known that the structure and physical properties of thin films grown in a glow discharge system strongly depend on the spatial distribution of the energy deposited by the particles that arrive at the substrate [42, 43]. Moreno-Marín et al. [42] have shown that during their travel through the plasma, the atoms reflected at the cathode lose less energy than the sputtered ones, so the former play a much more significant role than the latter in the deposition of energy at the substrate. According to the work of these authors, the number of sputtered atoms that land onto the substrate is appreciably larger than the number of reflected plasma particles, but the reflected particles carry the main part of the energy. This is a consequence of two important results. First, practically all high-energy particles emitted from the cathode are reflected gas particles and not sputtered atoms. Second, the transport through the plasma is advantageous to the high-energy reflected particles, so a significant number of sputtered particles will be thermalized in their travel to the substrate. Hence, the main part of the energy deposited onto the substrate is provided by the flux of reflected gas particles.

Based on the results described in the previous paragraph, we can consider the influence that the introduction of oxygen has on the structure of our films. Oxygen has a much lower atomic mass than argon. Hence, oxygen ions are much more mobile than argon. In the electric field of the sheath region of the plasma they will be accelerated to higher velocities than the argon ions, and, consequently, they will be reflected at the cathode with higher velocity too. The smaller volume and higher velocity of the reflected oxygen atoms will reduce their scattering probability by plasma species as they cross the plasma region. As a consequence, they will deposit more energy onto the substrate. A simple analogy with solid state physics will help to understand this. It is well known that the scattering of electrons by ionized impurities in a solid decreases with temperature. Elevating the temperature increases the thermal velocity of the carriers, which in turn reduces the time a carrier spends in the vicinity of an ionized impurity center. Hence, the smaller the deflection, the smaller the effect of the scattering event, and the greater the value of the mobility. An analogous effect is expected to reduce the scattering of the reflected oxygen ion, since their higher velocity compared to argon ions will make them spend less time in the vicinity of ionized scattering centers in the plasma. Additionally, the smaller volume of the oxygen ions reduces its 
scattering cross-section with both ionized and non-ionized centers. So the flux of reflected plasma particles in the case of oxygen plasma is expected to deposit more energy onto the substrate than in the case of argon plasma, thus promoting the growth of polycrystalline films in the first case while films grown with pure argon plasma remain amorphous.

In order to confirm experimentally the above hypothesis we have performed a simple experiment. We have measured the substrate temperature as a function of time when the substrate is not deliberately heated. Figure 6 shows the result for an oxygen plasma and an argon plasma, with all the other system parameters being the same (60 $\mathrm{W}$ of rf power and 1.2 mbar of gas pressure). A temperature of $127^{\circ} \mathrm{C}$ is reached with the oxygen plasma after 3000 $\mathrm{s}$ of deposition, while in the case of the argon plasma the temperature only reaches $75{ }^{\circ} \mathrm{C}$ in the same time. So it becomes clear that oxygen is depositing significantly higher energy onto the substrate.

However, microstructure is not only determined by particle bombardment. The surface and near-surface environment during film growth are crucial because they determine the adatom mobility. And the main parameters that control the ad-atom mobility are substrate temperature and particle bombardment [44]. As a function of these parameters, the microstructure of sputtered films is usually classified in terms of three zones [44, 45]. Zone 1 structure occurs when the ratio of growth temperature $(T)$ to melting point $\left(T_{m}\right)$ of the deposited material is less than 0.1. This is our case, because the melting point of hafnium oxide is $2774{ }^{\circ} \mathrm{C}$ [46], while the substrate temperature is $200{ }^{\circ} \mathrm{C}$, so our $T / T_{m}$ is 0.07 . In this range of growth temperatures ad-atom surface mobility is negligible, so in the case that a polycrystalline growth is promoted (in our experiment, by the high-energy reflected oxygen atoms) the initial nuclei tend to grow in the direction of the available incoming flux. The growth morphology is affected significantly by intergrain shading, so tapered crystallites develop and intergrain boundaries are voids rather than true grain boundaries. Hence, the film becomes underdense, although individual crystallites have near bulk density. Additionally, there is evidence that residual gas adsorption can reduce the movement of ad-atoms, so zone 1 structure is also promoted by the presence of impurities such as oxygen. Our composition measurements by ERDA have confirmed an excess of oxygen in the films deposited with oxygen or mixed oxygen/argon plasmas, while films deposited with pure argon are almost stoichiometric. So the presence of these oxygen impurities can play a role to explain the underdense structure of the polycrystalline films.

The index of refraction shown in Figure 4 confirms the above discussion about void formation and underdense structure in the polycrystalline film. The commonly reported value 
of refractive index for the bulk material is 2.08 at $632.8 \mathrm{~nm}$ [41]. In comparison our amorphous sample has an index of 1.98 at that wavelength, while the polycrystalline value is 1.68. The atomic density measurements performed by ERDA permit to calculate the mass density $(\rho)$ if the thickness of the films is known. The following equation is applied for this purpose:

$$
\rho\left(\mathrm{g} / \mathrm{cm}^{3}\right)=\frac{N \cdot d\left(\mathrm{at} / \mathrm{cm}^{2}\right)}{d(\mathrm{~cm})} \cdot \frac{\sum_{i}\left[A_{i}\right] M_{i}(\mathrm{~g} / \mathrm{mol})}{N_{A}(\mathrm{at} / \mathrm{mol})},
$$

where $N \times d$ is the ERDA areal density, $d$ is the thickness, $\left[\mathrm{A}_{\mathrm{i}}\right]$ and $\mathrm{M}_{\mathrm{i}}$ are the atomic per cent concentration and the atomic mass, respectively, of the i-th element detected by ERDA, and $N_{\mathrm{A}}$ is the Avogadro's number. For the polycrystalline sample deposited in oxygen plasma the ERDA areal density is $1.62 \times 10^{17} \mathrm{~cm}^{-2}$, while for the amorphous film this value is $1.20 \times 10^{17}$ $\mathrm{cm}^{-2}$. However, the amorphous sample is significantly thinner due to a slower deposition rate. According to the TEM values given in the experimental section, the thickness of the amorphous film is $10.9 \mathrm{~nm}$ versus $20.6 \mathrm{~nm}$ for the polycrystalline one. The atomic per cent concentration for each element, obtained from the ERDA analysis, are given in Table 1. The Hf and O concentrations add about $90 \%$ of the atomic content in both cases, although the stoichiometry (O/Hf ratio) is slightly higher in film deposited with oxygen plasma (2.20 versus 2.06 for the film grown with argon). The main impurity is hydrogen (between 7.4 and 8.1 at.\%), while other light elements are present in much smaller concentrations. $\mathrm{Zr}$ is detected in both cases because it is a common impurity in the Hf cathode, while Ar is detected only in the film deposited with argon plasma. By applying Equation 4 with the atomic concentrations given in Table 1 and the respective atomic masses, a mass density of $8.0 \pm 0.8$ $\mathrm{g} / \mathrm{cm}^{3}$ is obtained for the polycrystalline sample and $11.3 \pm 1.0 \mathrm{~g} / \mathrm{cm}^{3}$ for the amorphous one. This confirms the lower density of the polycrystalline film, in agreement with the lower value of the refractive index, and it is consistent with our previous discussion of void formation during growth of the polycrystalline structure.

There are very few references for the density of $\mathrm{HfO}_{2}$ films in the published literature. Generally, the value of a bulk $\mathrm{HfO}_{2}$ ceramic cubic crystal given by Ref. [46] and the theoretical value of $10.1 \mathrm{~g} / \mathrm{cm}^{3}$ determined from XRD are the most commonly cited values, but they are not useful for establishing a comparison with thin films that have a different structure (amorphous or monoclinic polycrystalline). For this purpose, the most useful previous study is probably the work of Teren et al. [47]. In this work the authors determined the areal mass of $\mathrm{HfO}_{2}$ films by X-ray fluorescence spectroscopy (XRF). To do this, they 
needed to perform an absolute calibration by Rutherford backscattering spectroscopy on films of different thickness ranging from $5 \mathrm{~nm}$ to $150 \mathrm{~nm}$. Within this range of thickness they were able to find the expected linear behavior between the XRF count-rate and the areal mass density of Hf. They also did additional determinations of thickness and density by X-ray reflectivity (XRR), using the relation between density and critical incidence angle for total reflection [48], and found consistent values. In general, an increase of the density of the films with deposition temperature and with annealing temperature was found in this study, which the authors attributed to crystallization and reduction in carbon impurity content. Low deposition temperatures produced amorphous films with densities in the range $5-8 \mathrm{~g} / \mathrm{cm}^{3}$, which increased to almost the bulk theoretical value of $10.1 \mathrm{~g} / \mathrm{cm}^{3}$ for deposition temperatures above the crystallization temperature of $500{ }^{\circ} \mathrm{C}$. The exception were some deposition conditions that create an oxygen deficiency at high deposition temperatures and therefore result in low density values. In our case, no oxygen deficiency is detected by ERDA, so the low value of density for the polycrystalline sample cannot be attributed to this cause. Our films have been grown at a low deposition temperature $\left(200^{\circ} \mathrm{C}\right)$, so the polycrystalline growth is not accompanied by thermal ordering and densification. On the contrary, the zone 1 structural growth discussed before is characterized by low surface ad-atom mobility and shadowing effects that create void space between the growing crystallites, and hence reduce the density. Finally, it should be noted that the amorphous films that have low density values (5-8 $\left.\mathrm{g} / \mathrm{cm}^{3}\right)$ in the study of Teren et al. have a content of carbon impurities between 5 and 7 $\%$. When this carbon is released by post-deposition annealing the films undergo relaxation and densification at temperatures lower than the crystallization temperature. As a function of deposition temperature, a high carbon content is also associated with a low density. In comparison, our amorphous film deposited with argon plasma has a negligible carbon content of less than 1 at.\%, and there are no significant amounts of other impurities that could affect its density by displacing $\mathrm{Hf}$ or $\mathrm{O}$ atoms. Hence, its amorphous structure is not an obstacle for a high density, as we observe in the ERDA data.

The higher value of the absorption coefficient for the polycrystalline sample in the transparency region has been discussed already in the results section in terms of the scattering of light by random reflections at crystallites boundaries. We will consider now the absorption coefficient in the region above the absorption edge. In the experimental section we introduced the two laws that usually fit the absorption coefficient in this region: Equation 1 for a direct band gap (a plot of $(\alpha h v)^{2}$ versus $h v$ should yield a straight line in this case) and Equation 2 (Tauc law) for an indirect band gap (when a plot of $(\alpha h v)^{1 / 2}$ versus $h v$ is a straight line). Both 
types of plots are shown in Figure 7. Comparing Figure 7a and Figure 7b it becomes apparent that most of the absorption region fits to a Tauc law characteristic of an indirect band gap (Figure 7a). The linear parts in the $(\alpha h v)^{2}$ versus $h v$ plot (Figure $7 \mathrm{~b}$ ) are limited to only few data points, and attempts to fit them to a linear regression resulted in linear correlation coefficients significantly lower than those obtained from the linear fits shown in Figure 7a. We conclude, therefore, that both types of film present an indirect band gap, whose values can be obtained from the fittings to the Tauc law and are $E_{G}=5.54 \pm 0.23 \mathrm{eV}$ for the amorphous film and $E_{G}=5.53 \pm 0.41 \mathrm{eV}$ for the polycrystalline sample. Hence, no significant differences appear in the band gap between the two types of films. This result confirms similar observations investigated with a different analytical method [26].

However, the Tauc coefficient, i.e. the slope of the plots in Figure 7a, was significantly different for the amorphous sample $\left(B_{\text {Tauc }}=1499 \pm 31 \mathrm{eV}^{-1 / 2} \mathrm{~cm}^{-1 / 2}\right.$ ) and the polycrystalline one $\left(B_{\text {Tauc }}=1344 \pm 49 \mathrm{eV}^{-1 / 2} \mathrm{~cm}^{-1 / 2}\right)$. That means the slope of the Tauc plot is steeper in the amorphous case, (i.e., the absorption coefficient increases faster in these films). In some studies, specially with amorphous solids, the Tauc plot has been related to the degree of order in the structure [29]. It is commonly admitted that disorder generates defects, and these in turn remove states from the bands and generate band tails of localized states. These band tails are generally described by the Urbach exponential law given by Equation 3 . Therefore, in a logarithmic plot of the absorption coefficient, the Urbach tail will appear as a linear region at energies below the absorption edge. The inverse of the slope of a linear fit to this region is the Urbach energy $E_{0}$.

An inverse correlation between the Tauc coefficient and the Urbach energy has been reported previously by us and other authors for different materials [29, 49]. It is based on the relation mentioned above between band states and band tailing. An example is the defect generation produced by the clustering that occurs around the percolation threshold of Si-Si bonds at a certain stoichiometry in hydrogenated silicon nitride [49]. In this example, the rupture of the percolation of the Si-Si bond network and the subsequent clustering, produces a strong tailing of band edges. As a consequence, energy levels are removed from the bands of extended states and a broad valence-band tail is formed, causing a minimum of the Tauc slope (less absorption above the absorption edge) and a maximum of the Urbach energy (lower slope in the region below the absorption edge due to the broader valence-band tail) [29, 49]. An analogous behavior can be postulated for the hafnium oxide films of the present study. When the structure of the films is amorphous (films deposited with pure Ar plasma), they are 
more dense and homogeneous, and have a higher value of the Tauc parameter, which is indicative of a uniform bond network with percolation of bond chains. On the contrary, when Zone 1 polycrystalline growth is promoted by the higher-energy reflected $\mathrm{O}$ atoms, percolation disappears and clustering occurs around the initial crystallites nuclei. Hence, a strong tailing of band edges appears, so the Tauc parameter decreases and an Urbach tail is developed. Both effects can be seen in Figures 5 and 7a. A linear plot to the Urbach tail of the polycrystalline sample has been performed in Figure 5 and results in an Urbach energy of 1.4 $\mathrm{eV}$.

Similar tailing of the absorption coefficient has been described before by Y. J. Cho et al. [17] for results of spectroscopic ellipsometry measurements on $16 \mathrm{~nm}$ thick hafnium oxide films deposited by reactive dc magnetron sputtering. These authors found a shift of the absorption edge to a higher energy and a decrease of band tailing as the samples were annealed at increasingly higher temperatures, which they attributed to defect reduction and temperature induced crystallization. However they did not fit the absorption curves to Tauc and Urbach laws to find $E_{\mathrm{G}}, B_{\text {Tauc, }}$ and $E_{0}$, so a comparison with our results is not straightforward. Other authors (Takeuchi et al. [18]) have also studied the effects of temperature on the absorption coefficient of $\mathrm{HfO}_{2}$ films characterized by spectroscopic ellipsometry. In this case the films were grown by rapid thermal oxidation of hafnium and the absorption coefficient was fitted by a Tauc law to obtain the value of the band gap. As the oxidation temperature was increased from $500{ }^{\circ} \mathrm{C}$ to $600{ }^{\circ} \mathrm{C}$ the band gap increased from 5.49 $\mathrm{eV}$ to $5.72 \mathrm{eV}$. The authors attributed this increase to a reduction of defects in the band-tail region. Those values of band gap match well with our results and with other published values obtained by vacuum ultraviolet absorption (5.8 eV) [50] and theoretical calculations $(6.0 \mathrm{eV})$ [51]. At lower absorption energies (below the absorption edge of the band gap) Takeuchi et al. observed an additional absorption peak in the range 4.5-5.0 eV. They interpreted this peak as an evidence of electron transitions from the valence band to available states within the band gap. This extra absorption was reduced when the oxidation annealing temperature was increased. These authors also examined the effect of the detected defects on the electrical characteristics (leakage current and $C$ - $V$ curves) and found that they act as electron traps. The origin of these traps was attributed to oxygen vacancies in the $\mathrm{HfO}_{2}$ film, which are known to produce electronic states in the band gap in many metal oxides [52]. Due to the method of film growth used by these authors (rapid thermal oxidation of a pure Hf film), the density of oxygen vacancies is likely to be high enough to influence the absorption spectra when insufficiently oxidized films with less oxygen than stoichiometric $\mathrm{HfO}_{2}$ are obtained. 
However, this is not our case, since our composition measurements by ERDA resulted in an $\mathrm{O} / \mathrm{Hf}$ ratio of 2.20 for the film deposited with oxygen plasma and 2.06 for the film grown with argon. Hence, the defects in our films are mostly associated with broken bonds at the surfaces of the polycrystalline grains rather than oxygen vacancies, so they are only responsible of the band tailing observed for the polycrystalline sample and they do not produce any absorption peak inside the band gap. Since our polycrystalline samples are a result of energy deposition on the substrate by high-energy reflected oxygen plasma particles and not the consequence of thermally induced ordering, it is not surprising that in our case defect related band tailing is more pronounced for the polycrystalline sample than for the amorphous one.

To end this discussion it is convenient to consider the influence that the different structure of the films has on their electrical characteristics, so that significant consequences can be drawn for their application as gate oxide in MOS technology. For this purpose we have performed quasistatic and high frequency capacitance measurements, whose results can be seen on Figure 8a. The most important parameter that can be extracted from these curves is the density of interface states (Figure 8b), which is related to the dip of the quasistatic curve. A lower density of traps at the interface means that the depletion region will extend deeper into the semiconductor as the bias is swept from accumulation to inversion, so the dip of the quasistatic capacitance will be more pronounced. This is the observed behavior for the amorphous film deposited with argon plasma compared to the polycrystalline sample grown with oxygen, which indicates that the amorphous structure forms an interface with a lower density of defects. Such observation is in agreement with the results discussed in the previous paragraphs, which suggested that an oxygen plasma deposits significantly more energy than an argon plasma onto the substrate during film growth, hence causing more damage to the interface.

The shift of the high frequency curve of the polycrystalline films towards negative gate voltages (Figure 8a) is indicative of a shift in the flat band voltage. A more negative flat band voltage means that there is more negative charge stored in the dielectric. This is also in agreement with the discussion of the results of the structural and optical measurements, where it was concluded that the polycrystalline samples are formed by individual crystallites with void intergrain boundaries that contain a high density of defects. Such defects trap charge and are responsible of the flat band voltage shift. In comparison, the amorphous samples were proposed to have a continuous and more dense bond network and hence a lower density of defects, which is corroborated by the lower trapped charge density. 


\section{Conclusions.}

We have described the optical and structural properties of $\mathrm{HfO}_{2}$ thin films and discussed how these properties are affected by the choice of glow discharge gas used for deposition. We have arrived to the conclusion that the introduction of oxygen into the argon glow discharge promotes zone 1 polycrystalline growth, while the structure of the films deposited with a pure argon plasma is amorphous. Zone 1 structure is a consequence of negligible ad-atom diffusion and shadowing effects, that result in the sputtered flux being deposited on high points on the film, with little material reaching the valleys. This leads to clustering in the form of tapered columns, with significant voids between clusters. As a consequence, polycrystalline films are underdense, they have a lower refractive index and show a strong tailing in the absorption edge, while amorphous films have a smooth, compact and homogeneous structure. It has been shown that these morphological and optical differences between the two kinds of films have an important influence on the electrical characteristics of the material, and therefore on its application to devices.

\section{Acknowledgements}

Parts of this work were done during a research stay of one of the authors (F.L.M.) at the Ionen Strahl Labor (ISL) of the Hahn-Meitner-Institut (HMI) with support of a mobility grant (PR2004-0426) of the Spanish Ministry of Education and Science. It was also supported by the research project TEC2004/1237 and the research grant (AP2003-4434) of the same Ministry. Special thanks are given to the ISL and to the CAI-"Tecnologías Físicas" for a long trajectory of fruitful collaboration. 
Table 1. Atomic per cent concentration of each element detected by ERDA in the films deposited with oxygen or argon plasma

The uncertainty of the O/Hf ratio is about $5 \%$

\begin{tabular}{ccccccccccc}
\hline Elements & Hf & O & H & B & C & N & F & Ar & Zr & O/Hf ratio \\
\hline Film with O plasma (at.\%) & 28.5 & 62.6 & 7.4 & 0.27 & 0.35 & 0.05 & 0.63 & 0 & 0.2 & 2.2 \\
Film with Ar plasma (at.\%) & 29.2 & 60.3 & 8.1 & 0.05 & 0.8 & 0.2 & 0.15 & 1 & 0.2 & 2.06 \\
\hline
\end{tabular}


Figure 1. XRD pattern for a sample deposited with oxygen plasma (upper trace) and a sample deposited with argon plasma (lower trace). Peaks corresponding to monoclinic $\mathrm{HfO}_{2}$ are marked by vertical lines.

Figure 2. Infrared spectra showing peaks associated with $\mathrm{HfO}_{2}$ for a sample deposited with oxygen plasma (upper trace) and a sample deposited with argon plasma (lower trace).

Figure 3. Transmittance and reflectance spectra of an amorphous and a polycrystalline sample.

Figure 4. Index of refraction of the amorphous and polycrystalline films.

Figure 5. Absorption spectra for the amorphous and polycrystalline $\mathrm{HfO}_{2}$ films. The straight line is a linear fit to the Urbach tail (see discussion).

Figure 6. Substrate temperature versus time for an oxygen and an argon plasma when the substrate is not deliberately heated.

Figure 7. Tauc plot (part A) and dependency of $(\alpha h v)^{2}$ on photon energy (part B). From part (A) of the figure the optical gap is obtained by fitting the absorption coefficient to the Tauc law. The corresponding linear fits are shown.

Figure 8. Part $\mathrm{A}$ of this figure shows high frequency $\left(C_{\mathrm{HF}}\right)$ and quasistatic $\left(C_{\mathrm{Q}}\right) C-V$ characteristics for an amorphous sample deposited in argon plasma (continuous trace) and a polycrystalline film grown with oxygen plasma (dashed line). On part B, the corresponding distributions of density of interface states $\left(D_{\mathrm{it}}\right)$ are plotted versus trap energy $\left(E_{\mathrm{t}}\right)$ measured with respect to conduction band edge $\left(E_{\mathrm{C}}\right)$. 
FIGURE 1

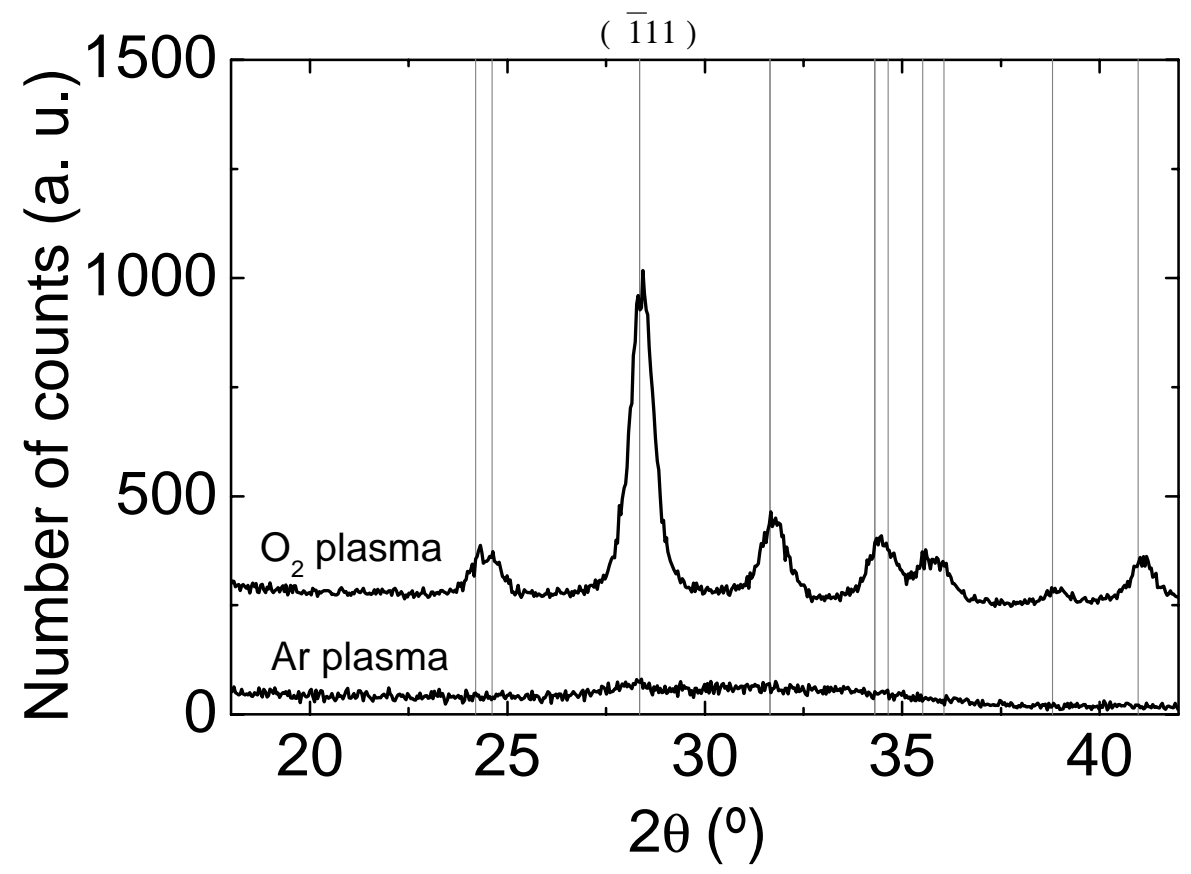


FIGURE 2

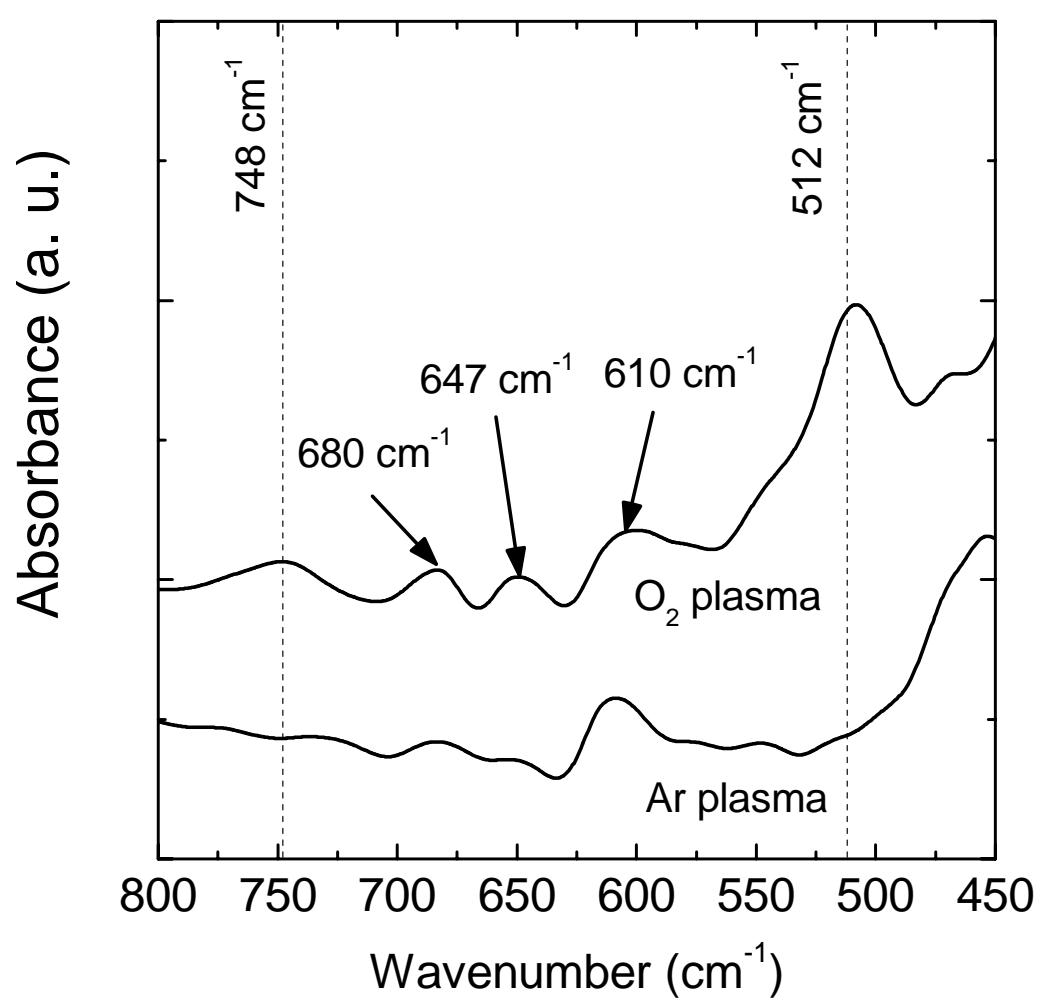


FIGURE 3

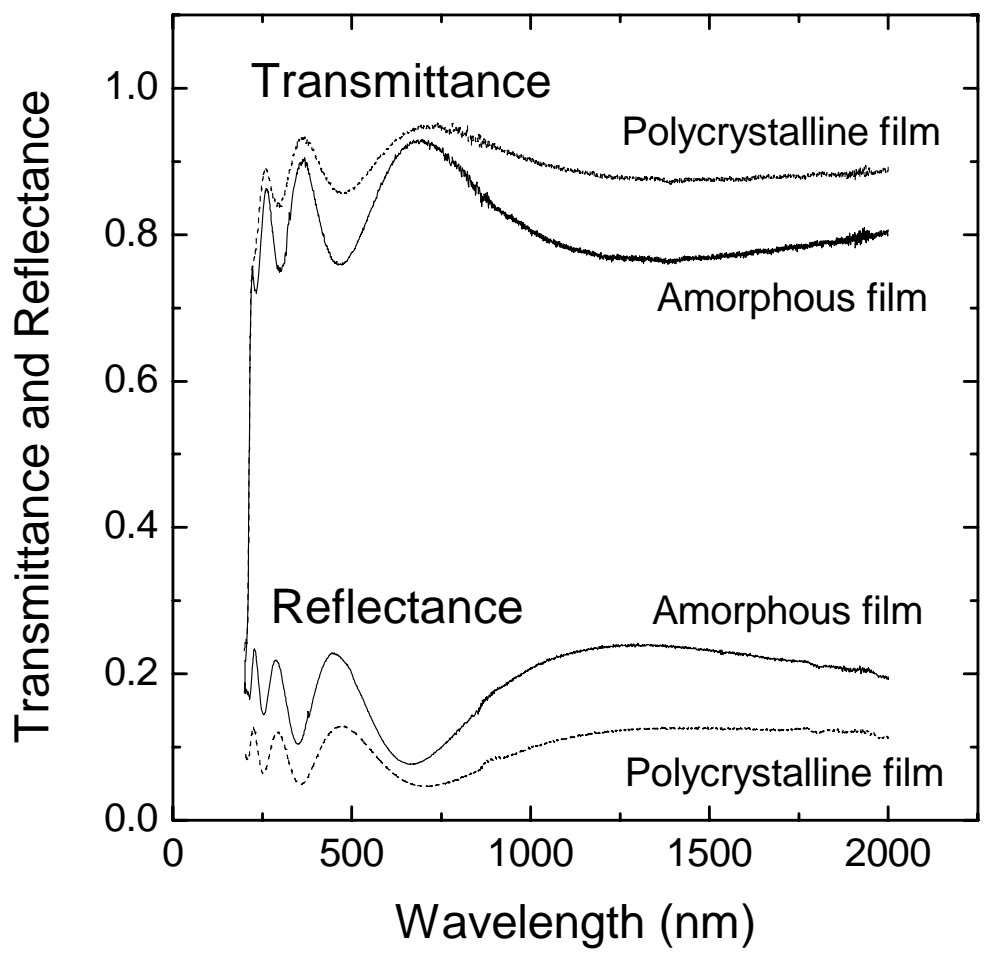


FIGURE 4

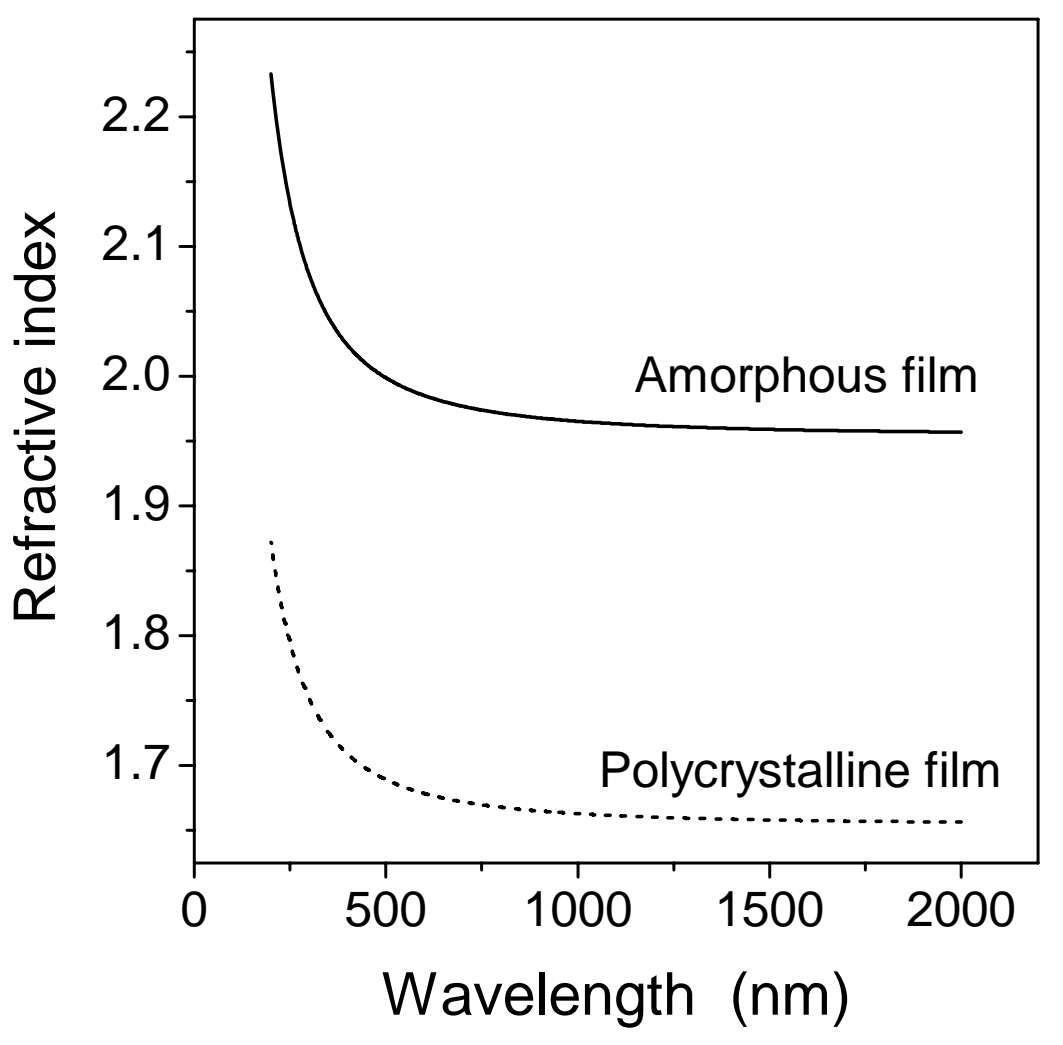


FIGURE 5

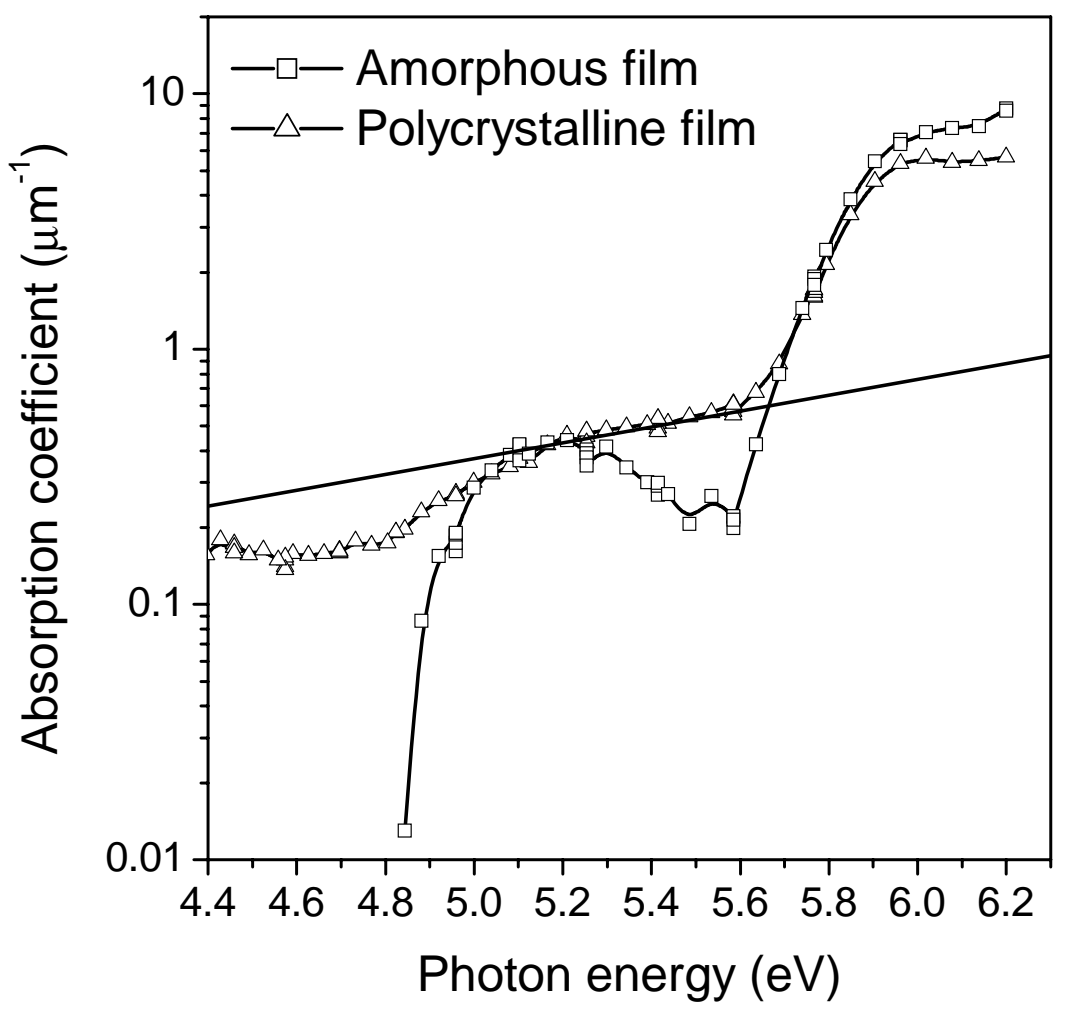


FIGURE 6

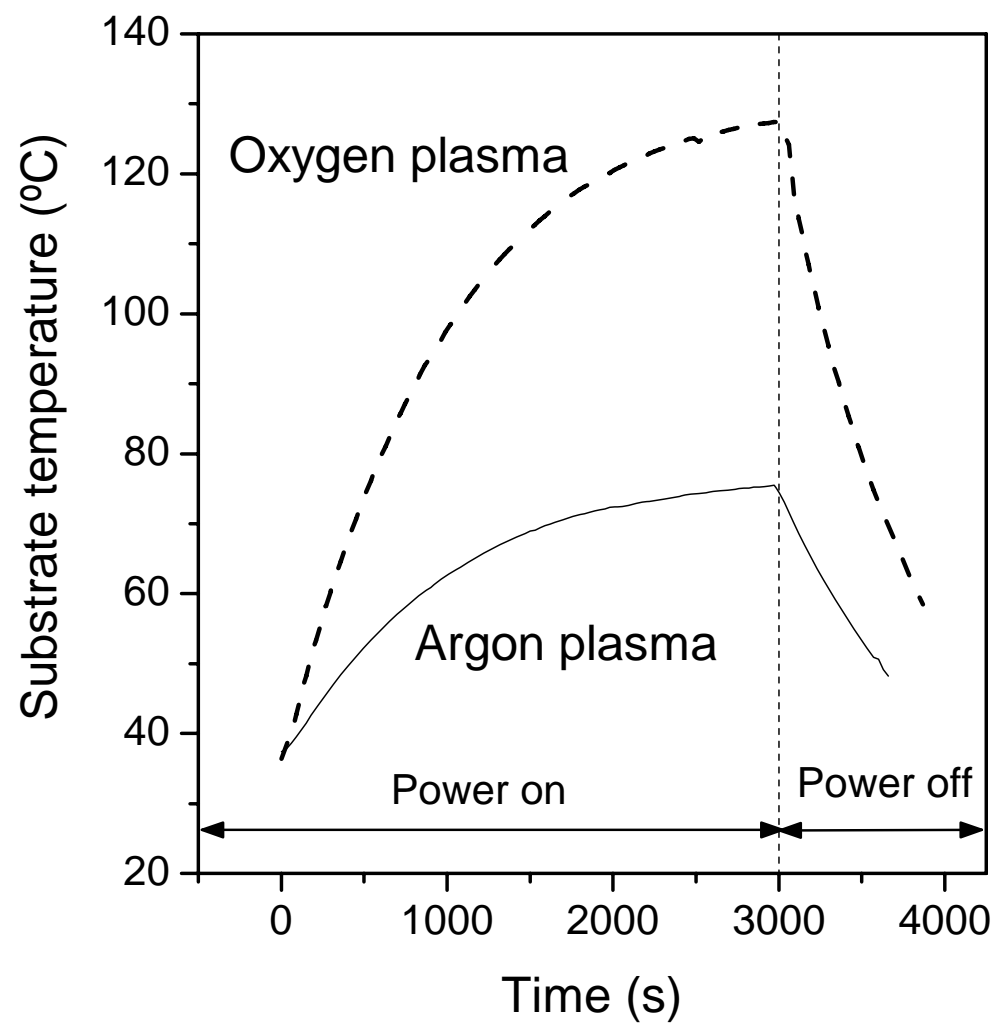


FIGURE 7A

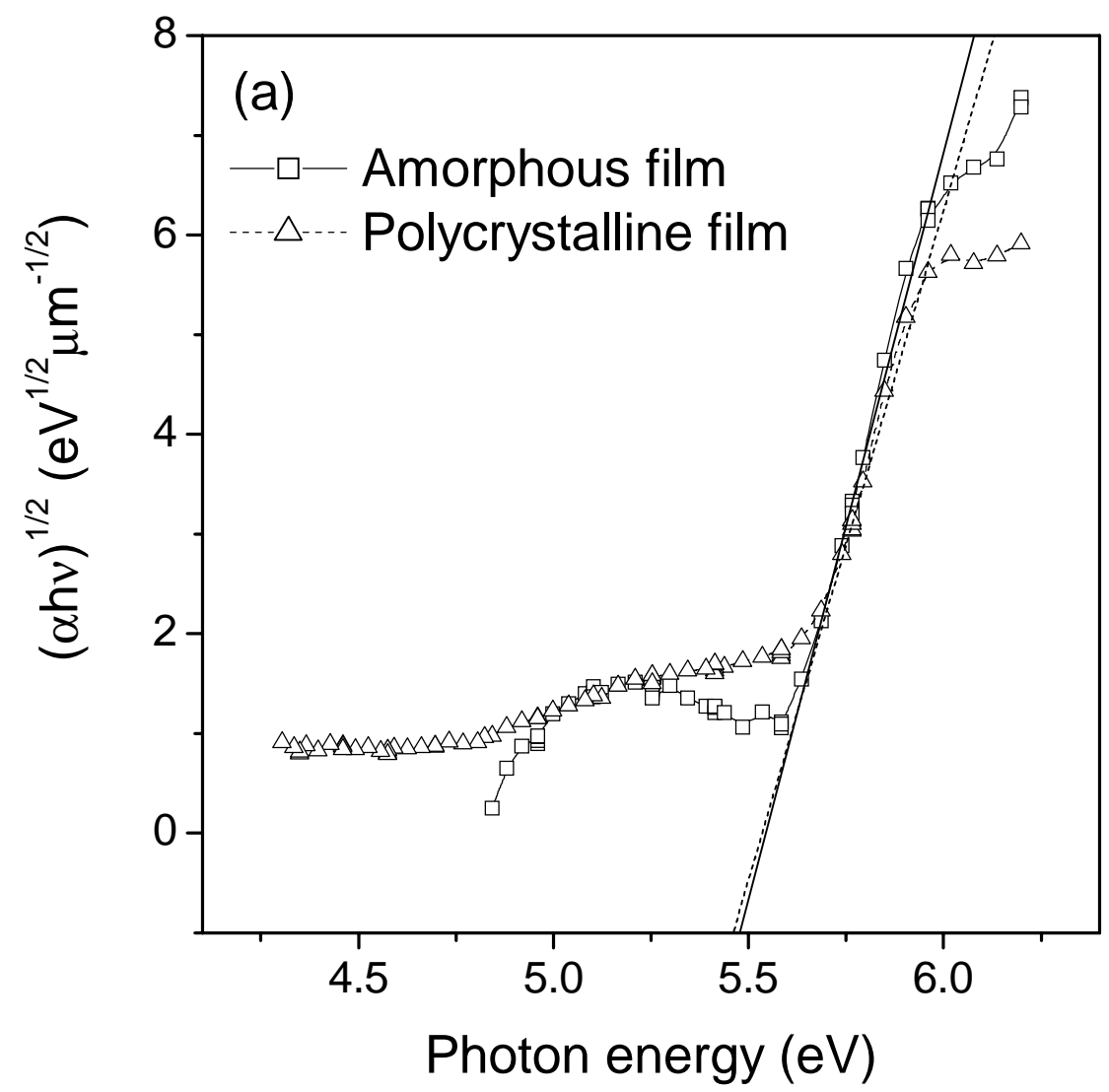


FIGURE 7B

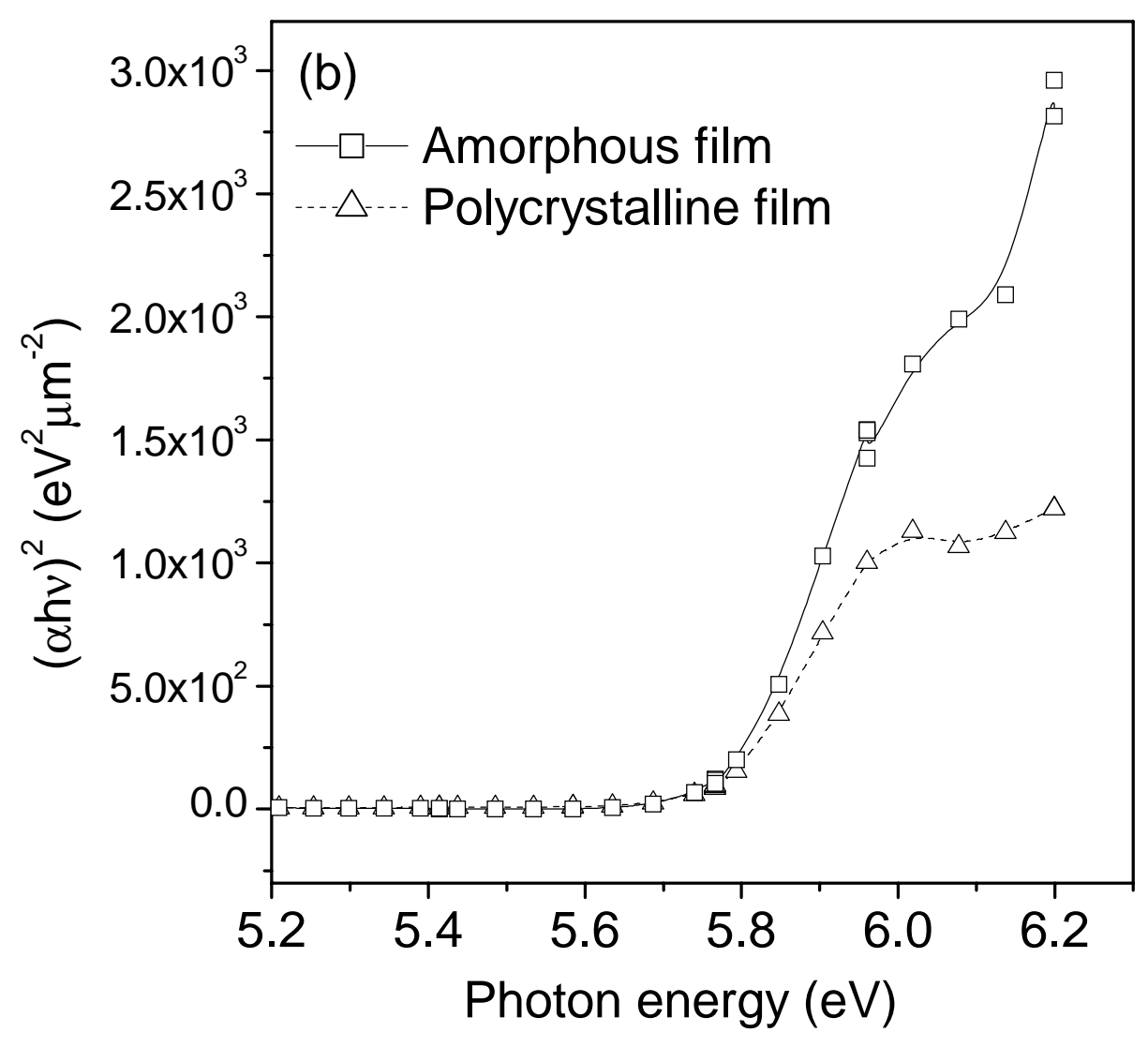


FIGURE 8A

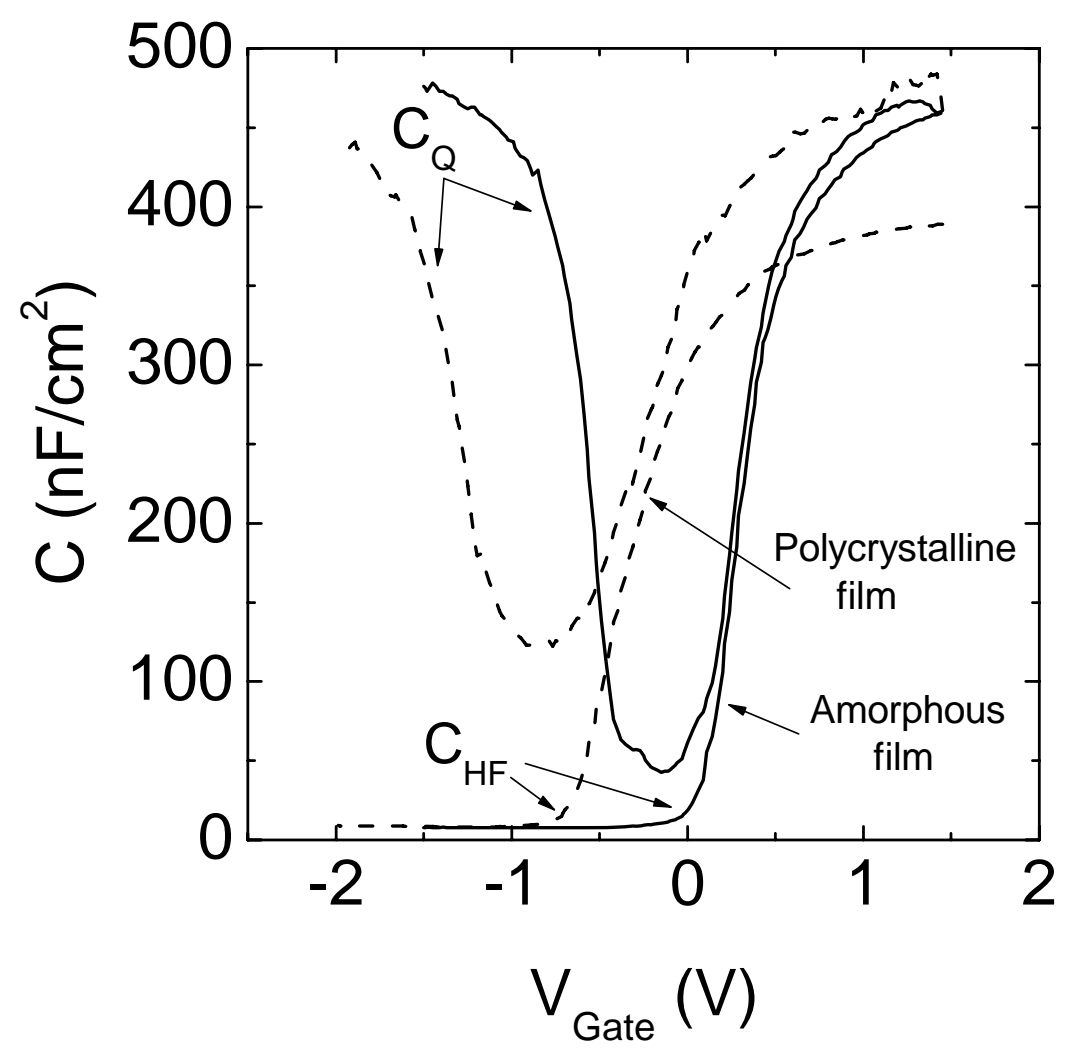


FIGURE 8B

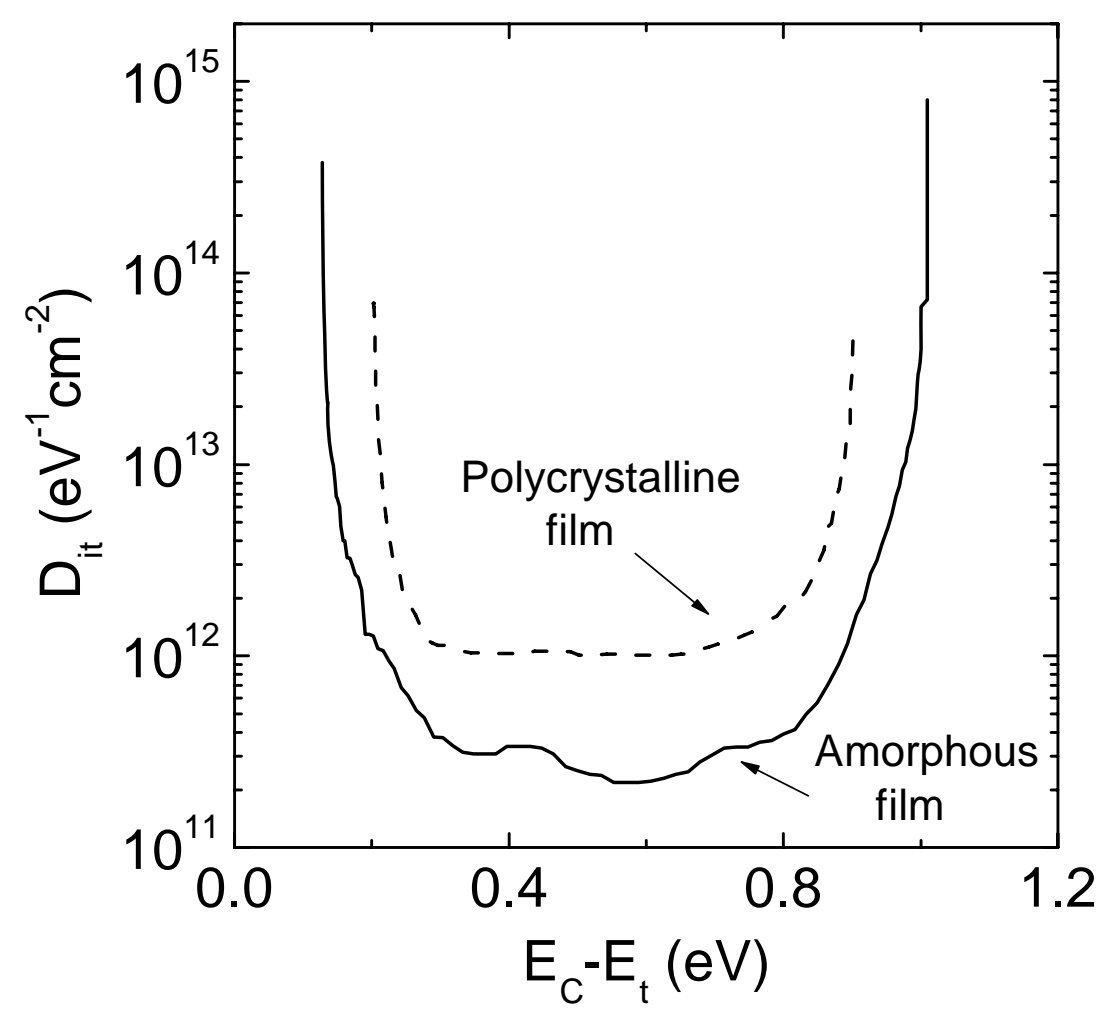




\section{References}

${ }^{1}$ Wilk G D, Wallace R M, and Anthony J M, 2001 J. Appl. Phys. 89, 5243.

${ }^{2}$ Kingdom A I, Maria J P, and Streiffer S K, 2000 Nature 406, 1032.

${ }^{3}$ Martínez F L, del Prado A, Mártil I, González-Díaz G, Bohne W, Fuhs W, Röhrich J, Selle B, and Sieber I, 2001 Phys. Rev. B 63, 245320.

${ }^{4}$ Martínez F L, Ruiz-Merino R, del Prado A, San Andrés E, Mártil I, González-Díaz G, Jeynes C, Barradas N P, Wang L, and Reehal H S, 2004 Thin Solid Films 459, 203.

${ }^{5}$ Mártil I, del Prado A, San Andrés E, González-Díaz G, and Martínez F L, 2003 J. Appl. Phys. 94, 2642.

${ }^{6}$ San Andrés E, del Prado A, Mártil I, González-Díaz G, and Martínez F L, 2003 J. Vac. Sci. Technol. 21, 1306.

${ }^{7}$ del Prado A, San Andrés E, Mártil I, González-Díaz G, Bravo D, López F J, Fernández M, and Martínez F L, 2003 J. Appl. Phys. 94, 1019.

${ }^{8}$ del Prado A, San Andrés E, Mártil I, González-Díaz G, Bravo D, López F J, Bohne W, Röhrich J, Selle B, and Martínez F L, 2003 J. Appl. Phys. 93, 8930.

${ }^{9}$ del Prado A, San Andrés E, Martínez F L, Mártil I, González-Díaz G, Bohne W, Röhrich J, Selle B, and Fernández M, 2002 Vacuum 67, 507.

${ }^{10}$ Buchanan D A, 1999 IBM J. Res. Develop. 43, 245.

${ }^{11}$ San Andrés E, Pantisano L, Severi S, Trojman L, Ferain I, Toledano-Luque M, Jurczak M, Groeseneken G, De Gendt S, and Heyns M, Microelectronic Engineering, in press.

${ }^{12}$ Toledano-Luque M, Pantisano L, Degraeve R, Zahid M B, Ferain I, San Andrés E, Groeseneken G, and De Gendt S, Microelectronic Engineering, in press.

${ }^{13}$ Robertson J, 2006 Rep. Prog. Phys. 69, 327.

${ }^{14}$ Poppe U, Klein N, Dhäne U, Soltner H, Jia C L, Kabius B, Urban J, Lubig A, Schmidt K, Hansen S, Orbach S, Müller G, and Piel H, 1992 J. Appl. Phys. 71, 5572.

${ }^{15}$ San Andrés E, Toledano-Luque M, del Prado A, Navacerrada M A, Mártil I, González-Díaz G, Bohne W, and Röhrich J, 2005 J. Vac. Sci. Technol. A 23, 1523.

${ }^{16}$ Saxena A N and Mittal K L, 1975 J. Appl. Phys. 46, 2788.

${ }^{17}$ Cho Y J, Nguyen N V, Richter C A, Ehrstein J R, Lee B H, and Lee J C, 2002 Appl. Phys. Lett. 80, 1249.

${ }^{18}$ Takeuchi H, Han D, and King T-J, 2004 J. Vac. Sci. Technolol. A 22, 1337.

${ }^{19}$ Zhao X and Vanderbilt D, 2002 Phys. Rev. B 65, 233106.

${ }^{20}$ Anastassakis E, Papanicolaou P, and Asher I M, 1975 J. Phys. Chem. Solids 36, 667. 
${ }^{21}$ Carlone C, 1992 Phys. Rev. B 45, 2079.

${ }^{22}$ Hirata T, 1994 Phys. Rev. B 50, 2874.

${ }^{23}$ Neumayer D A and Cartier E, 2001 J. Appl. Phys. 90, 1801.

${ }^{24}$ Lesser M, 1987 Optical Engineering 26, 911.

${ }^{25}$ Fadel M, Azim O A, Omer O A, and Basily R R,1998 Appl. Phys. A 66, 335.

${ }^{26}$ Aarik J, Mändar H, Kirm M, and Pung L, 2004 Thin Solid Films 466, 41.

${ }^{27}$ Callegari A, Cartier E, Gribelyuk M, Okorn-Schmidt J F, and Zabel T, 2001 J. Appl. Phys. 90, 6466.

${ }^{28}$ Hernández-Rojas J L, Lucía M L, Mártil I, González-Díaz G, Santamaría J, and SánchezQuesada F, 1992 Appl. Opt. 31, 1606.

${ }^{29}$ Martínez F L, del Prado A, Mártil I, González-Díaz G, Selle B, and Sieber I, 1999 J. Appl. Phys. 86, 2055.

${ }^{30}$ Kern W and Puotinen D, 1970 RCA Review 31, 187.

${ }^{31}$ Martínez F L, Toledano M, San Andrés E, Mártil I, González-Díaz G, Bohne W, Röhrich J, and Strub E, 2006 Thin Solid Films 515, 695.

${ }^{32}$ Toledano-Luque M, San Andrés E, Olea J, del Prado A, Mártil I, Bohne W, Röhrich J, and Strub E, 2006 Mat. Sci. Semicond. Proc. 9, 1020.

${ }^{33}$ Bohne W, Röhrich J, and Röschert G, 1998 Nucl. Instr. and Meth. B 136-138, 633.

${ }^{34}$ Bohne W, Fuhs W, Röhrich J, Selle B, González-Díaz G, Mártil I, Martínez F L, del Prado A, 2000 Surf. Interface Anal. 30, 534.

${ }^{35}$ Al-Kuhaili M F, 2004 Optical Materials 27, 383.

${ }^{36}$ Swanepoel R, 1983 J. Phys. E: Sci. Instrum. 16, 1214.

${ }^{37}$ Nicollian E H and Brews J R, 1982 MOS (Metal Oxide Semiconductor) Physics and Technology, (New York, John Wiley \& Sons).

${ }^{38}$ Hann R E, Suitch P R, and Pentecost J L, 1985 J. Amer. Ceram. Soc. 68, C-285.

${ }^{39}$ Cullity B D and Stock S R, 2001 Elements of X-ray diffraction, (Upper Saddle River: Prentice Hall).

${ }^{40}$ Frank M M, Sayan S, Dörmann S, Emge T J, Wielunski L S, Garfunkel E, and Chabal Y J, 2004 Mater. Sci. Eng. B, 1096.

${ }^{41}$ Hu H, Zhu C, Lu Y F, Wu Y H, Liew T, Li M F, Cho B J, Choi W K, and Yakolev N, 2003 J. Appl. Phys. 94, 551.

${ }^{42}$ Moreno-Marín J C, Abril I, and García-Molina R, 1999 J. Vac. Sci. Technol. A 17, 528.

${ }^{43}$ Winters H F, Coufal H J, and Eckstein W, 1993 J. Vac. Sci. Technol. A 11, 657. 
${ }^{44}$ Vossen J L and Kern W, 1991 Thin Film Processes II, (San Diego: Academic Press).

45 Thornton J A, 1974 J. Vac. Sci. Technol. 11, 666.

${ }^{46}$ Weast R C, Astle M J, and Beyer W H (Eds.), 1987 CRC Handbook of Chemistry and Physics, (Boca Ratón: CRC Press).

${ }^{47}$ Teren A R, Thomas R, He J, and Ehrhart P, 2005 Thin Solid Films 478, 206.

${ }^{48}$ Lengeler B, in: Campagna M and Rosei R (Eds), 1990 Photoemission and Absorption

Spectroscopy with Synchrotron Radiation, (Amsterdam: North Holland).

${ }^{49}$ Robertson J, 1994 Philos. Mag. B 69, 307.

${ }^{50}$ Kato H, Nango T, Miyagawa T, Katagiri T, Seol K S, and Ohki Y, 2002 J. Appl. Phys. 92, 1106.

${ }^{51}$ Robertson J, 2000 J. Vac. Sci. Technol. B 18, 1785.

${ }^{52}$ Morant C, Fernández A, González-Elipe A R, Soriano L, Stampfl A, Bradshaw A M, and Sanz A M, 1995 Phys. Rev. B 52, 11711. 